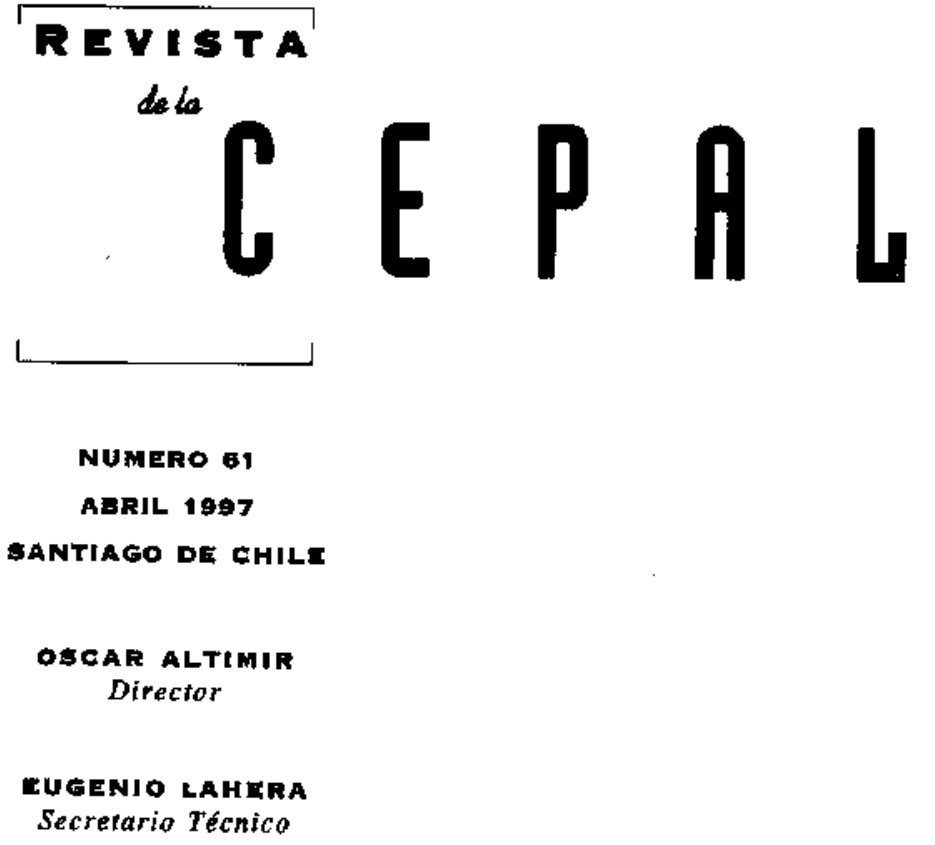

Secretario Técnico

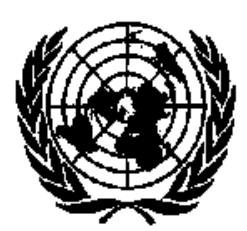

NACIONES UNIOAS 
Tres formas de coordinación social

Norbert Lechner

Brechas sociales en Colombia

Juan Luis Londoño de la Cuesta

Los Estados Unidos al rescate: la asistencia flnanciera a México en 1982 y 1995

Nora Lustig

El réglmen de convertibllidad y el sistema bancario en la Argentina Alfredo F. Calcagno

Exportaciones de manufacturas: desafíos para las pequeñas economías de América Latina

Rudolf M. Buitelaar, Pitou van Dijck

¿Por qué las inversiones en el transporte público no reducen la congestión de tránsito urbano?

lan Thomson

Nolas sobre la mediclón de la pobreza según el método del ingreso

Juan Carlos Feres

La politica fiscal y el ciclo económico en Chile

Carlos Budnevich, Guillermo Le Fort

Evaluación de la política de bienes de capital en la Argentina

Pablo Sirlin

Reestructuraclón de los grupos industriales brasileños entre 1980 y 1993

Ricardo $M$. Ruiz

Reestructuración productiva y cambio territorial: un segundo eje de Industrlallzación en el norte de México

Tito Alegría, Jorge Carrillo, Jorge Alonso Estrada

Publicaciones recientes de la CEPAL 


\section{Notas sobre la medición de la pobreza según el método del ingreso}

\section{Juan Carlos Feres}

Division de Estadística y Proyecciones Economicas, CEPAL
Los diferentes resultados que suelen obtener diversos estudios que se abocan a la medición de la pobreza en un país, al parecer con el mismo método y las mismas fuentes de datos, desn conciertan a los analistas y a la opinión pública en general. Las discrepancias - sobre la magnitud del fenómeno y las características de los hogares considetados pobres- restan credibilidad y confiabilidad técnica a estas mediciones, ponen en tela de juicio las evaluaciones del nivel y la evolución de la pobreza y dificultan las comparaciones internacionales. De allí la importancia de propiciar un mayor consenso entre los investigadores respecto a los criterios y procedimientos que se aplican, a fin de encaminarse hacia un patron común que haga más consistentes y homogéneas las mediciones y garantice su efectiva comparabilidad. En este artfculo se reseñan algunos aspectos del denominado "método del ingreso" que inciden en la identificación de los hogares pobres y en el cálculo de la extensión de la pobreza, y que por ser útiles al propósito señalado debieran merecer una consideración especial en las futuras investigaciones sobre el tema. Ellos son: las diferencias que se establecen en el costo de la canasta básica de alimentos; el procedimiento de cálculo del valor de los satisfactores no alimentarios; el uso de medidas de equivalencia para los hogares de diferente tamafío y composición; la evaluación de la confiabilidad de la medición del ingreso corriente; los problemas asociados a la ampliación de la cobertura del concepto de ingreso; las medidas de la pobreza y, finalmente, las fuentes de información normalmente utilizadas en este tipo de estudios. 
I

\section{Introducción}

En América Latina se utilizan preferentemente dos aproximaciones metodológicas para medir y caracterizat la pobreza: el llamado "método del ingreso" o de las "líneas de pobreza" y el método directo de los indicadores sociales, cuya modalidad más difundida en los últimos años ha sido la de los denominados "mapas de necesidades básicas insatisfechas".

Como se sabe, ambos métodos responden a enfoques conceptuales diferentes, al punto de que "no constituyen, en realidad, formas alternativas de medir la misma cosa, sino que representan dos concepciones distintas de la pobreza" (Sen, 1981); 'estas concepciones se basan, en un caso, en la noción de la capacidad para satisfacer las necesidades esenciales y, en el otro -il método directo-, en la observación del consumo real de las personas en relacion a determinadas convenciones sobre necesidades mínimas. Sin embargo, los dos métodos revisten gran interés y contribuyen significativamente a las tareas de diagnostico de la pobreza.

La fuente principal de información de los mapas de necesidades básicas insatisfechas son los censos de población y vivienda, en tanto que las estimaciones por las líneas de pobreza se elaboran sobre la base de las encuestas de hogares. En ambos casos se cuenta, naturalmente, con un esquema metodologico y una cierta matriz operacional. No obstante, la aplicación concreta de ellos suele transitar por variantes que no siempre se explican del todo por las consabidas limitaciones en materia de información. Esto lleva a que con alguna frecuencia los resultados de diversos estudios, que utilizan aparentemente el mismo método y las mismas fuentes de datos, en especial bajo el enfoque de las líneas de pobreza, difteran en la magnitud de la pobreza estimada (a veces en medida considerable) y en las características de los hogares que son clasifican dos como pobres, para evidente desconcierto de los analistas y la opinión pública en general. Tales discrepancias inciden en la credibilidad y confiabilidad técnica de estas mediciones, ponen en tela de juicio las evaluaciones del nivel y evolución de la pobreza y dificultan las comparaciones internacionales.

Los esfuerzos por encarar este problema pasan, obviamente, por cuidar en cada investigación la transparencia y acuciosidad con que se explican los concep- tos, criterios y supuestos utilizados, así como las fuentes de informacion y el tratamiento a que se someten los datos, de manera de hacer posible un riguroso examen e interpretación de los resultados.

Pero esto no basta. Al mismo tiempo, es preciso generar entre los investigadores un mayor consenso en cuanto a los procedimientos que se aplican en las diferentes etapas del proceso de estimación, a fin de que se encaminen hacia un patrón común capaz de aumentar la consistencia y homogeneidad de las mediciones y garantizar su efectiva comparabilidad. Lo cual se complementa, desde luego, con una indagación aún más profunda sobre la verdadera utilidad, alcances y limitaciones de la información que se obtiene con estos métodos, en función de lo que se necesita para el análisis y la toma de decisiones en el campo social (diagnóstico y definición de grupos focales, así como diseño, seguimiento y evaluación del impacto de las políticas y programas orientados a la superación de la pobreza).

Aunque esto forma parte de un debate ya tradicional en América Latina, y bastante se ha avanzado al respecto, creemos que es posible ampliar todavía más el espacio de aplicación común de conceptos y métodos; sin perjuicio de que por la naturaleza y complejidad del tema se deba admitir siempre -e incluso se estime aconsejable - cierto grado de diversidad.

En este artfculo se reseñan brevemente algunos aspectos del método del ingreso, de carácter exclusivamente metodológico, que inciden en la identificación de los hogares pobres y el cálculo de la extensión de la pobreza, y que pueden ser útiles a tal propósito. Su importancia ya ha sido puesta de manifiesto en la vasta literatura que sobre el particular se ha generado en la región en los últimos años, por lo que debieran merecer una especial consideración en las investigaciones futuras sobre el tema.

Centraremos los comentarios en torno a siete elementos: las diferencias que se establecen en el costo de la canasta básica de alimentos; el procedimiento de cálculo del valor de los satisfactores no alimentarios; el uso de medidas de equivalencia para los hogares de diferente tamaño y composición; la evaluación de la confiabilidad de la medición del ingreso corriente; los problemas asociados a la ampliación de la cobertura 
del concepto de ingreso; las medidas de la pobreza y, finalmente, algunos alcances sobre las fuentes de in formación que se emplean en este tipo de estudios.

No se abordan, por lo tanto, en esta oportunidad los aspectos relativos a la dimensión propiamente con- ceptual del método del ingreso, lo que no significa desconocer que ella tiene gran importancia y que, en ocasiones, puede condicionar fuertemente las opciones en el plano metodológico.

\section{II}

\section{Canasta básica de alimentos}

La determinación del valor de la canasta básica de alimentos, representativo del costo de satisfacer las necesidades básicas de alimentación en un momento y lugar determinados, es un proceso de varias etapas que demanda gran cantidad de información. En América Latina esa tarea se ha asumido pritscipalmente en el marco de lo que podría describirse como los lineamientos de la cEPAl: la definición de canastas cuyo nivel y composición satisfagan las necesidades nutricionales y reflejen los hábitos de consumo prevalecientes en la sociedad (ajuste a las preferencias del consumidor), en concordancia con la oferta interna de productos alimentarios y sus precios relativos. Para la valoración de la canasta se utilizan los precios de cada artículo recolectados para el cálculo del índice de precios al consumidor (CEPAL, 1991)

Pese a que en la región esta metodología se aplica de manera relativamente generalizada, y que ella detalla los procedimientos que ordenan las diferentes fases de la estimación, cada ejercicio concreto, por insuficiencias de la información u otras razones, se enfrenta a situaciones particulares, que al final influyen en el costo de la canasta básica de alimentos. Asf́, es casi inevitable que se llegue a valores diferentes, no solo entre distintos investigadores sino inchuso en el contexto de un mismo estudio, según las opciones adoptadas en cuanto a criterios, supuestos y tratamiento de los datos búsicos. A su vez, los distintos valores de la canasta básica de alimentos, conocidos también como líneas de indigencia o de pobreza extrema, se traducen directamente en diferencias en la dimension estimada de la pobreza.

El problema es todavía más agudo cuando la información con que se cuenta es manifiestamente débil o incompleta, como suele ocurrir respecto de algunas áreas urbanas o las áreas rurales de los países de la región, lo que obliga a apoyar el análisis en meras hipó tesis o conjeturas.
Ahora bien, no obstante estar conscientes de ello, por lo general tendemos a trabajar, implícita o explícitamente, bajo el supuesto de que el costo de la canasta básica de alimentos representa un valor específico, de alta precisión. Y lo mismo hacemos luego, por ende, con el valor del índice de pobreza.

En atención a la variabilidad descrita, ${ }^{1}$ pareccría más razonable establecer este costo en términos de un intervalo y no considerarlo necesariamente un valor especffico. La contrapartida obvia es que de ese modo se dificulta un tanto el análisis de los cambios, sobre todo de los pequeños, que experimenta la pobreza en el tiempo; pero lo que se gana es una mayor coherencia con el grado de exactitud con que efectivamente se obtienen estas estimaciones. Puesto así, el problema sería entonces cómo determinar la amplitud del citado intervalo.

La tarea es, sin duda, eminentemente empírica. Dejando de lado los aspectos netamente estadísticos, los mínimos y máximos del intervalo debieran ser la resultante de la sensibilidad del costo de la canasta básica de alimentos a valores alternativos, dentro de rangos razonables, de ciertos parámetros relevantes que intervienen en su estimación. Entre los factores de mayor incidencia cabe mencionar aquellos que guardan relación i) con los requerimientos nutricionales de los diferentes grupos (según sexo-edad y actividad), dado que la canasta se ajusta a las necesidades medias de la población; ii) con la estructura de consumo implícita en la canasta básica de alimentos y la cantidad física de cada producto que la compone, y iii) con los precios a los cuales se valora dicha canasta.

\footnotetext{
${ }^{1}$ A la cual se sunia la variabilídad estadística propia del caracter mestral de algunos datos que se utilizan en la construcción de la canasta básica de alimentos, como la distribución socioxlemográfica $o$ las estructuras de consume.
} 
Los primeros incluyen, entre otros, la talla y por consiguiente el peso de las personas adultas, necesarios para calcular la tasa de metabolismo basal (energía) o el requerimiento proteínico; la distribución horaria entre las diferentes actividades predominantes (ligeras, moderadas y pesadas), especialmente en la población adulta, y el gasto energético bruto correspondiente a cada actividad. Entre los segundos están los aspectos relativos a la selección del grupo de referencia para evaluar los hábitos de la población; al tratamiento del consumo "fuera del hogar" (modalidad de adquisición de los alimentos), y a los supuestos sobre la evolución de los patrones de gasto, cuando no se dispone de encuestas recientes sobre el particular. $\mathbf{Y}$ entre los terceros se encuentran la selección de los precios que se estimen pertinentes para valorar la canasta básica de alimentos (precios medios, mínimos, los que pagan los sectores pobres, etc.); las diferencias entre regiones o áreas, a falta de información detallada, y el índice válido para actualizar el valor de la canasta (FFC de los alimentos, IPC de los pobres o precios por producto).

Diversos estudios (CEPAL, 1991; Gerstenfeld, 1993) han simulado el efecto de algunos de estos factores. Por ejemplo, se ha analizado la sensibilidad de las necesidades medias de energía de toda la población a las variaciones de sus elementos determinantes. Así, cambios en la talla de la población adulta de más o menos $2 \mathrm{~cm}$ conducen a variaciones - positivas o negativas-- en las necesidades calóricas de no más de $22 \mathrm{Kcal}$ al día por persona (menos de $1 \%$ del requerimiento calorico total). Las distintas hipótesis sobre la distribucion de horas entre las diferentes actividades predominantes, por su parte, causan variaciones de no más de $26 \mathrm{Kcal}$ al día por persona. $\mathrm{X}$ el impacto de los diversos valores de los gastos energéticos brutos correspondientes a cada actividad se mueve entre $-1.2 \%$ y $0.7 \%{ }^{2}$ Del mismo modo, en ciertos países y para determinados perfodos, las diferencias en el costo de la canasta básica de alimentos derivadas de utilizar uno u otro indice de precios para actualizar su valor han resultado mínimas o de poca significación.

No obstante, la información de que se dispone es aún muy insuficiente y no siempre aporta resultados concluyentes. Debe hacerse más por alcanzar cierta formalización que permita establecer algo así como el efecto neto del conjunto de factores que inciden en las variaciones del valor de esa canasta. Este es, por tanto, uno de los temas en que se debe seguir trabajando.

\section{III}

\section{Gastos no alimentarios}

En el marco del método de las líneas de pobreza, la manera habitual de determinar el costo de satisfacción de las necesidades no alimentarias es de tipo normativo. Se basa en la relación observada entre los gastos en alimentación y los gastos totales de consumo (coeficiente de Engel) en los distintos estratos de hogares, especialmente en aquellos que pertenecen al denominado grupo de referencia (hogares cuyo gasto en alimentos es ligeramente superior al presupuesto básico establecido). ${ }^{3}$

\footnotetext{
${ }^{2}$ Se debe tener presente, sin embargo, que si las variaciones se expresaran en relación a los requerimientos de las personas aduttas, en lugar del promedio de toda la población, estos porcentajes serian algo superiores a los indicados.

${ }^{3}$ Quizás si la única excepción a este procedimiento la constituya el trabajo "Macroeconomía de las necesidades esenciales en México" (COPlamar, 1983), en el que se hizo un intento por identificar satisfactores especificos para cada tipo de necesidad no alimentaria.Véase también Boltvinik, 1990.
}

Al respecto, se han destacado a lo menos dos connotaciones conceptuales. En primer lugar, lo anterior supone que los hogares que logran cubrir adecuadamente sus necesidades de alimentacion satisfacen, al mismo tiempo, los estándares mínimos de las otras necesidades básicas. ${ }^{4} \mathrm{En}$ segundo lugar, el hecho de que el dato registrado en las encuestas corresponda exclusivamente al gasto en consumo privado significa que una parte de la satisfacción de las necesidades básicas no queda reflejada en esa pauta de consumo, en la medida en que los hogares acceden a bienes y servicios subsidiados total o parcialmente por el Estado (CEPAL, 1990). ${ }^{5}$

\footnotetext{
4 Esto no es necesariamente así. Sobre el particular, véase en Beccaria y Minujin (1993) un interesante análisis del tema de la vivienda, con miras al tratamiento de los ingresos imputados por concepto de uso de la vivienda propia.

${ }^{5}$ Esto se retoma más adelante en la sección VI.
} 
Sin embargo, lo que queremos resaltar acá es un asunto distinto. Admitido el coeficiente de Engel como norma a partir de la cual se establece el costo de los satisfactores no alimentarios, surge el problema de las diferencias en el valor de este coeficiente entre los distintos tipos de hogares de un estrato de referencia. ${ }^{6}$ Tales diferencias tienen que ver principalmente con el tamaño y la composición del hogar y con la etapa del ciclo de vida por la que éste atraviesa.

Un ejemplo de ello lo encontramos en los resultados del análisis efectuado por Feres y León (1988) a partir de los datos de la encuesta de ingresos y gastos de Colombia de 1984-1985, que ilustran acerca de las diferencias que existen en la estructura del gasto no alimentario, en términos de sus principales rubros, entre hogares de similar nivel de ingreso pero de distinta composición. Así, en el conjunto de hogares de Bogota, Cali y Medellín que conformaban el segundo cuartil de gasto per cápita se observaron diferencias en la proporción del gasto destinado a transporte, educación, salud y vivienda. Respecto de este último rubro, los hogares constituidos por parejas jóvenes sin hijos destinaban en promedio cerca de $20 \%$ del gasto a vivienda, en tanto que en los hogares de parejas de mayor edad con dos hijos la cifra era de $15 \%$. Las diferencias que se observaron en la proporcion del gasto dedicado a la alimentación fueron aún mayores.

Así, puesto que el costo de atender las necesidades no alimentarias varía a lo largo de las etapas del ciclo de vida familiar según el tamaño y la composición del hogar y también en función de factores como la clantía del patrimonio acumulado y el grado de acceso a los servicios públicos, se justifica un análisis pormenorizado de estos capítulos del gasto, que podría derivar -al igual que para la alimentación- en eventuales coeficientes específicos para distintos tipos de hogares.

Sin embargo, este es un aspecto que debiera también tener su expresión en el ámbito de las denominadas "escalas de equivalencias" que se analizan en la sección IV; tal vez a través de ellas debieran recoger. se completamente los efectos de las diferencias de composición y las economías de escala de los hogares implícitas en el perfil de sus gastos de consumo.

Por otra parte, en virtud de la baja periodicidad con que se realizan las encuestas de presupuestos familiares, es necesario contar con algún criterio que permita actualizar la estructura de gastos de los hoga* res. En ausencia de otra información, y sin recurrit a sofisticados cálculos de elasticidad, una vía disponible parece ser la de considerar la evolución de los distintos capítulos del gasto y ponderar, en cada momento, el coeficiente original por las diferencias de los cambios en los precios relativos. La experiencia de los últimos años en la mayoría de los países de la región en cuanto, por ejemplo, a la disparidad con que se han modificado los precios de los bienes transables y los de los bienes no transables, o las tarifas de los servicios públicos frente a los precios de los alimentos, justifica con creces un procedimiento así.

Un asunto distinto (y que no veremos acá) es, por cierto, la manera de determinar los coeficientes de gasto en alimentación en aquellos lugares para los cuales no se dispone de información, como sucede con gran parte de las zonas rurales de América Latina.

\section{IV}

\section{Medidas de equivalencia}

En los estudios de pobreza se utilizan normalmente las unidades per cápita para expresar tanto los valores de las líneas de pobreza como los recursos de que dispo-

\footnotetext{
${ }^{6}$ En los trabajos de la cEpal se han adoptado corrientemente, y de manera uniforme para todos los países, coeficientes de 0.5 para las zonas urbanas y de cerca de 0.57 para las zonas rurales, los que equivalen a valores inversos (relación de Orshansky) de 2.0 y 1.75 , respectivamente (Altimir, 1979). En algunos palses en que el coeficiente medio observado en el grupo de referencia se aparta de estos valores, ellos igualmente se han conservado con el objeto principal de no alterar la comparabilidad con estimaciones previas (CEPAL, 1991).
}

ne el hogar para satisfacer sus necesidades básicas, reconociéndose asi el que el nivel de bienestar depende estrechamente del número de miembros de la familia. Sin embargo, como ya se dijo antes, en rigor las líneas de pobreza, o el ingreso, debieran reconocer además las economías de escala asociadas a diferentes tamaños de hogares, los efectos de la particular composición de sus integrantes (por sexo, edad u otra característica relevante), aś como la estructura de consumo que acompaña a las distintas fases o etapas del ciclo de vida familiar. El conjunto de estos elementos es 10 
que tendría que resumirse en las denominadas "escalas de equivalencia" entre los hogares.

La utilización o no de este tipo de escalas en los diversos estudios redunda en resultados que discrepan no sólo en cuanto a la extensión de la pobreza sino, especialmente, en la identificación de los hogares pobres. Como ejemplo baste señalar que muchos hogar res de ingresos medios pero de gran tamaño ( $y$ por ende con fuerte presencia de niños) podrían cambiar su ciasificación de pobres a no pobres si se tomaran en cuenta las equivalencias que les son aplicables.

No obstante, debe reconocerse que estas escalas son difíciles de obtener, y aunque existen algunas propuestas concretas para mejorarlas, se considera que hasta ahora son muy parciales e imperfectas. Tres son las aproximaciones principales que se utilizan para elaborar dichas escalas: i) las encuestas de valoración de las necesidades individuales (introduce elementos subjetivos); ii) la investigación empírica del comportamiento del gasto de los hogares (usando algún indicador de bienestar, como el coeficiente de Engel, para hogares de diferente tamaño y composición), y los estudios basados en información nutricional (y psicológica).

Naturalmente, los distintos métodos conducen a resultados también distintos.

Para ilustrar la gran disparidad que se puede producir a este respecto, en el cuadro 1 se presentan cinco escalas actualmente en uso en diferentes países. Como se advierte, ellas pueden llegar a exhibir grandes diferencias, al punto de alterar significativamente los resultados que se obtengan en un estudio de pobreza. De modo que antes de decidir entre aplicar o no aplicar una escala es muy importante conocerlas a fondo y evaluar sus antecedentes y racionalidad.

Un criterio utilizado en América Latina para elaborar escalas de equivalencia, pero válido tan sólo para el consumo alimentario, es aquel que se basa en las necesidades energéticas de cada individuo (CEPAL, 1991). Aunque con ello es posible tomar en cuenta las diferentes necesidades de alimentación de los distintos miembros del hogar, no incorpora explícitamente las posibles economías de escala en el consumo de alimentos. Por lo tanto, no se puede considerar este criterio como suficiente para extrapolarlo al conjunto de los gastos de consumo, aunque su utilidad es indudable en el rubro alimentario. En el cuadro 2 se pre-

7 Y todavía se puede agregar otro factor, cuyo análisis empírico resulta aún más complicado: la distribución intrahogar del consumo. senta un ejemplo de este tipo de escala, a partir del requerimiento energético de un hombre adulto de 31 a 60 años de edad con actividad moderada.

CUADRO I

Escalas de equivalencia entre los hogares

\begin{tabular}{lcc}
\hline & Hombre & Mujer \\
\hline A. Escala de Amsterdani & & \\
18 afios y más & 1.00 & 0.90 \\
14 a I7 affos & 0.98 & 0.90 \\
Menos de 14 ainos & 0.52 & 0.52
\end{tabular}

B. Escala de la Orzanización de Cooperación y Desarrollo Económicos (OCDE) $)^{b}$

Primer adulto

Persona adicional de 14 afios o más

Persona adicional menor de 14 affos

C. Escala de la ocDE modificadac

Primer adulto

Persona adicional de 14 aftos o más

Persona adicional menor de 14 años

D. Escala subjetiva

Primer adulto

Segunda persona

Tercera persona

Cuarta persona

Quinta personta

Sexta persona

exra persona

E. Escala LIs ${ }^{\mathrm{d}}$

Jefe de hogar

Adultos y niños

Usada en los estudios de gasto de consurno en el Reino Unido.

b Arbitraria, pero más realista que la escala per ć́pita.

- Compromiso entre la escala de la oCDE y la escala subjetiva.

d LIS: Luxemburgo Income Study

CUADRO 2

Perú: Escala de equivalencia entre los hogares basada en las necesidades energéticas

\begin{tabular}{|c|c|c|c|}
\hline Categorias sociod & nográficas & Hombres & Mujeres \\
\hline Menores de 1 año & & 0.274 & 0,253 \\
\hline 1 a 3 años & & 0.502 & 0.469 \\
\hline 4 a 6 años & & $0.65 !$ & 0.587 \\
\hline 7 a 9 años & & 0.748 & 0.660 \\
\hline 10 a 13 afios & & 0.825 & 0.728 \\
\hline $\begin{array}{l}14 \text { a } 17 \text { antos } \\
18 \text { a } 30 \text { aftos }\end{array}$ & & 0.990 & 0.774 \\
\hline Actividad $=$ & ligera & 0.883 & 0.701 \\
\hline Actividad $\Leftarrow$ & moderada & 0,994 & 0.722 \\
\hline Actividad $\Leftarrow$ & pesada & 1.143 & $0.76 \mathrm{l}$ \\
\hline $\begin{array}{l}31 \text { a } 60 \text { años } \\
\text { Actividad } \Leftarrow\end{array}$ & ligera & 0.888 & 0.725 \\
\hline Aclividad $\Leftarrow$ & moderada & 1.000 & 0.747 \\
\hline Actividad $\doteqdot$ & pesada & 1.150 & 0.787 \\
\hline Mayores de 60 af & & & \\
\hline Actividad $\Longleftarrow$ & ligera & 0.729 & 0.659 \\
\hline Actividad $\Longleftarrow$ & moderada & 0.821 & 0.679 \\
\hline Actividad $\Longleftarrow$ & pesada & 0.944 & 0.715 \\
\hline
\end{tabular}

Fuente: CEPAL, 1991, anexo 2. 
En consecuencia, pese a reconocer la imperiosa necesidad de incorporar las escalas de equivalencia en la metodologia de los estudios de pobreza, es poco aún lo que se puede proponer ślidamente al respecto.
Constituye, por lo tanto, otro tema que debiera formar parte de la agenda de investigación metodológica en este campo para los países de la región.

\section{$\mathrm{V}$}

\section{Confiabilidad de la medición del ingreso}

Entre los múltiples aspectos que investigan las encuestas de hogares, no cabe duda de que uno de los más controvertidos es la calidad de las mediciones de las distintas corrientes de ingresos que reciben las personas y las familias. Se sabe que ellas presentan sesgos, tradicionalmente subestimativos, pero no hay igual consenso respecto a la magnitud de esos sesgos y la forma de determinarlos (Feres, 1988), En los estudios de pobreza, que representan un corte normativo en la distribución del ingreso, se deben evaluar estos sesgos y corregirlos. De lo contrario, ellos se reflejarán automáticamente en la dimensión estimada de la pobreza.

Pese a que las encuestas que se utilizan con frecuencia para estos fines forman parte -en casi todos los países- de un programa regular y de ejecución permanente, con contenidos y procesos altamente estandarizados, no hay antecedentes suficientes para avalar la tesis de una relativa estabilidad de estos sesgos en las sucesivas rondas de la encuesta. ${ }^{8}$ Por el contrario, en muchos casos la información que se ha podido generar tiende a contradecir, a veces abiertamente, la idea de un posible "congelamiento" de tales sesgos. No queda otra alternativa, por tanto, que evaluar en cada encuesta la confiabilidad de la medición del ingreso y tratar de corregir los márgenes de subestimación que se establezcan.

Los sesgos pueden ser de distinto tipo. Algunos se relacionan con el carácter muestral de la investigación (insuficiencia o mala calidad del marco muestral, problemas de cobertura, rechazos, variabilidad estadística, etc.), en tanto que otros derivan más bien de contingencias o errores ajenos al muestreo (Altimir, 1975). Los primeros se suponen manejados en el marco de cada encuesta y son generalmente evaluados por el

\footnotetext{
${ }^{8}$ De existir esa estabijidad, no se nesolvería el problema de la precisión de las estimaciones, pero si en buena medida el de su comparabilidad, lo que incidiría positivamente en los análisis de la distribución del ingreso.
}

responsable de elia. Los segundos, en cambio, constituyen sobre todo errores de respuesta que suelen ser más dificiles de detectar, que no siempre son completamente imputables al encuestado, cuya corrección resulta compleja y que suelen representar una proporción mayor del error total de estimación.

Para nuestros propositos interesan especialmente los problemas de no respuesta, de cobertura incompleta del concepto de ingreso investigado y de subdeclaración.

En las encuestas, la falta de respuesta a determinadas preguntas sobre el ingreso puede llegar a ser significativa, al punto de distorsionar los resultados y abultar artificialmente los índices de pobreza (y particularmente los de indigencia). ${ }^{9}$ Es necesario entonces efectuar las imputaciones correspondientes según los atributos de cada receptor que no declaró. Esto es perfectamente posible en la mayoría de los casos y la propia encuesta aporta información para ello, utilizan. do como base los ingresos declarados por las personas u hogares de similares características. ${ }^{10}$

Otro criterio alternativo es, obviamente, el de excluir de la muestra, para todo lo relacionado con las variables de ingreso, a quienes no informen de su valor. Si lo anterior se acompaña de los ajustes muestrales pertinentes (reemplazos a cambios en los factores de expansión) no habría problema; pero, de no ser así, la representatividad de la encuesta se alteraría o habría

\footnotetext{
${ }^{9}$ Un ejemplo extremo lo constituye la encuesta permanente de hogares (EPH) de Argentina. En la encuesta de la Capital Federal y el Gran Buenos Aires de octubre de 1990, el 23.2\% de los ocupados no reporto sus ingresos del trabajo. En 1992 dicho porcentaje fue de $17.8 \%$.

${ }^{10}$ En general, esto no presenta mayores problemas en las corrientes de ingreso provenientes del trabajo, jubilaciotes o pensiones y alquileres imputados (que constituyen alrededor del $90 \%$ de los ingresos totales). Naturalmente es más complicado en los restantes tipos de ingreso, donde es muy difícil detectar posibles onisiones. A la vez, existen probadas técnicas computacionales para efectuar estas imputaciones. Al respecto véase Feres, 1996.
} 
que ampararse en el supuesto de que las personas que se excluyen de la muestra tienen la misma distribución y características de las que permanecen en ella. Un supuesto como éste requiere' al menos del respaldo de investigaciones en el terreno, que por lo general no se realizan en Américà Latina.

El concepto de ingreso investigado en las encuestas también puede completarse por el mecanismo de la imputación a fin de compatibilizarlo con el ingreso corriente total de los hogares. Pero este caso es distinto al anterior. Acá no sólo es necesario identificar a los probables perceptores de cada clase de ingreso no investigado, sino además generar una estimación del monto global o promedio de esos ingresos a partir de datos de una fuente exógena a la encuesta que, como se verá, está constituida por las cuentas nacionales. Asimismo, se debe contar con algunos criterios que permitan distribuir esos ingresos entre los perceptores. En la práctica, lo que sucede es que a veces la encuesta entrega información que permite identificar a esos receptores y distribuir los ingresos, y otras veces sólo es posible hacer una asignacion burda y sobre la base de conjeturas. Sin embargo, aun en este caso es preferible realizar las correcciones, minimizando aś el riesgo de sobreestimar los índices de pobreza.

Por su parte, para el análisis de la confiabilidad de los datos sobre los ingresos y la evaluación de los probables sesgos de subdeclaración también se requiere un patrón đe referencia cuantitativo, independiente de la encuesta misma, que provea estimaciones de los diversos tipos de ingreso de los receptores y sirva como base de comparación. Este papel se le asigna normalmente a las cuentas nacionales.

Hasta ahora la mayoría de los países de América Latina no elabora regularmente la cuenta de ingresos y gastos de los hogares del sistema de cuentas nacionales, o no lo hace con el grado de desagregación deseado. A la vez, son de sobra conocidas las críticas que se formulan a la precisión y confiabilidad de las propias cuentas nacionales, lo que podría poner en tela de juicio su utilización como marco para evaluar las encuestas. No obstante, es indiscutible que se trata del único sistema estadístico que permite una evaluación y conciliación en detalle de los datos provenientes de múltiples fuentes, en un marco conceptual coherente y de aplicación sistemática.

De allí que lo importante sea más bien cómo acrecentar progresivamente la disponibilidad, calidad, nivel de desagregación y rigor conceptual de la cuenta de los hogares y no limitarse solamente a impugnar su validez. En esta línea se podrían inscribir, por ejemplo, los esfuerzos de la CEPAL por incentivar a los países a que asuman esta tarea, colaborando en el desa- rrollo metodológico e incluso aportando sus propias estimaciones.

El siguiente paso es definir los criterios y procedimientos para ajustar los datos sobre ingresos obtenidos en la encuesta. ${ }^{11} \mathrm{Si}$ suponemos que la magnitud de la subestimación de cada tipo de ingreso viene dada por su diferencia respecto al monto de similar corriente de ingreso registrada en las cuentas nacionales, la primera tarea que se debe enfrentar es la conciliación de conceptos entre ambas fuentes. Para ello es preciso considerar algunos que se examinan a continuación.

\section{Remuneraciones de los empleados}

De conformidad con los instructivos, las encuestas investigan los ingresos del trabajo y en particular las remuneraciones de los empleados, generalmente en términos líquidos (vale decir, lo que efectivamente recibe el trabajador luego de efectuados los descuentos legales). En la cuenta de los hogares, por su parte, se consignan las remuneraciones brutas, de manera que para lograr la equivalencia conceptual con los datos de la encuesta es preciso deducir de estas últimas las contribuciones a la seguridad social (para obtener remuneraciones netas) y los impuestos directos.

Esto es posible si se utiliza la información sobre el valor de las contribuciones al sistema de seguridad social. Las que se hacen al régimen tradicional (de reparto) se incluyen entre los gastos de los hogares, y las que se hacen al régimen de capitalización individual (de existir) deben estimarse especialmente como partidas informativas de la cuenta. ${ }^{12}$

\section{Excedente de explotación}

Del excedente de explotación se debe excluir aquel que se refiere al sector de propiedad de vivienda (arriendos efectivos e imputados), el que a su vez debiera ser objeto de un tratamiento separado en lo que toca a su comparación con la encuesta.

De esta forma, el excedente de explotación neto, excluido el del sector de propiedad de vivienda y deducidos los impuestos directos, se asimila al concepto de ingreso primario de los trabajadores independientes, cuya medición es la que se procura en las encuestas.

\footnotetext{
11 Véase una descripción detallada de los criterios utilizados por dicho organismo en sus estudios sobre la magnitud de la pobreza en CEPAL, 1991, cap. II.

${ }^{12}$ Más adelante, en el apartado 5 de esta sección, se comentan algunas dificuliades conceptuales y prácticas vinculadas al tratamiento contable de las transacciones de los nuevos sistemas previsionales.
} 


\section{Impuestos directos}

A las remuneraciones netas de los empleados registradas en la cuenta de los hogares es necesario todavía descontarles los impuestos directos, para hacerlas consistentes con el concepto de remuneraciones líquidas que presumiblemente captan las encuestas. Lo propio debe hacerse respecto de las ganancias, ya que el concepto contable también incluye tales impuestos.

Para ello hay que desglosar los impuestos directos pagados por los hogares y registrados en la cuenta, de manera de asociarlos (enteramente) a cada una de estas dos fuentes de ingreso. Con ese propósito es necesario sistematizar la información disponible en cada oportunidad sobre los diferentes tipos de impuesto, a fin de estimar el respectivo valor pagado por los sueldos y salarios y, por diferencia, deducir el correspondiente a las ganancias.

\section{Arriendos imputados y arriendos efectivos}

En cada uno de los afios para los que se estime la cuenta de los hogares se debe incluir un conjunto de partidas informativas. Entre ellas cabe contemplar los alquileres imputados por el uso de la vivienda propia o cedida, y la identificación de aquella parte del excedente de explotación del sector propiedad de vivienda que corresponde a arriendos imputados.

Estos datos permiten, naturalmente, disponer del valor de los arriendos imputados, pero además calcular el de los arriendos efectivos e introducir los mencionados ajustes al excedente de explotación para hacer consistente su comparación con el concepto de ganancia de los trabajadores independientes captado en las encuestas.

\section{a) Arriendos imputados}

Como se desprende de lo anterior, las partidas informativas correspondientes deben incluir tanto la produccion bruta de los arriendos imputados como su correspondiente valor agregado (excedente de explotación). La diferencia entre ambos se explica por los costos de producción, que en este caso se asocian básicamente a los gastos de reparaciones, las contribuciones de bienes raíces, el consumo de capital fijo y los gastos comunes. ${ }^{13}$

${ }^{13}$ Los valores estimados para algunos países revelan que la proporción que representan estos costos puede alcanzar niveles notablemente altos. En Chile, por ejemplo, éstos llegan al 46\% del valor de producción de los arriendos imputados (porcentaje determinado en la matriz de insumo-producto).
Ahora bien, aun cuando en términos del ingreso de los hogares en rigor correspondería hacer la comparación con los arriendos imputados que se obtienen de la encuesta a nivel de valor agregado, es razonable suponer que en la práctica los hogares entrevistados responden en función de lo que ellos estiman podría ser el valor de arriendo de su vivienda en el caso de que ella debiera contratarse en el mercado. De modo que el monto que declaran se aproxima más ał concepto de valor de producción que al de valor agregado, debido a que es dable presumir que los hogares no descuentan ninguno de los rubros de costo señalados anteriormente. Más aún, tampoco sería posible calcular estos costos sobre la base de alguna otra información registrada en la encuesta.

De allí entonces que, para esta corriente de ingreso no monetario, la comparación entre las magnitudes del marco de referencia y las de la encuesta se establezca normalmente a nivel del valor total de los arriendos imputados. Esto sin perjuicio de que en el curso de la comparación se presenten, además, otros problemas, a los cuales haremos referencia más adelante.

\section{b) Arriendos efectivos}

De acuerdo con la información disponible en las partidas informativas, esta corriente de ingreso se obtiene como la diferencia entre el excedente de explotación del sector propiedad de vivienda y aquel que corresponde a los arriendos imputados. Cuando en las encuestas se la investiga por separado, queda abierta la posibilidad de contrastar directamente la medición que de ella efectúan ambas fuentes de información.

\section{Prestaciones del nuevo sistema de seguridad social}

El valor de las prestaciones que reciben los hogares de los nuevos sistemas de seguridad social (de capitalización individual) en los países que los han aplicado, y que es incluido entre las partidas informativas, se contabiliza conjuntamente con el valor de las prestaciones del régimen antiguo de seguridad social que se registra en la cuenta de los hogares. Esto responde a la necesidad de hacer equivalente el concepto del marco de referencia con el efectivamente declarado en las encuestas.

Cabe señalar que las prestaciones del nuevo régimen no figuran entre los ingresos de la cuenta porque, desde el punto de vista contable, los aportes al nuevo sistema se consideran una transacción financiera (ahotro) y sus prestaciones se asimilan a un retiro de fon- 
dos propios (o desahorro). No obstante, al evaluar los recursos disponibles del hogar, y dada la modalidad que asumen normalmente estos retiros, parece más lógico considerarlos ingreso corriente.

De cualquier manera, es del caso recordar que éste es uno de los tipos de ingreso que para los efectos del ajuste se contabiliza en términos netos, ya que las cotizaciones son deducidas simultánearnente de las remuneraciones de los empleados.

Un tratamiento algo distinto es el que se aplica a las transacciones con las instituciones privadas de salud previsional, cuando ellas existen, en la medida en que se las asimila a las compañ́as de seguro. Contablemente, sus prestaciones se registran en el rubro de ingreso de los hogares bajo la partida de indemnización del seguro de riesgos, al tiempo que las cotizaciones de los empleados se incluyen como primas netas por ese seguro, $y$ una parte menor como gasto de consumo final, porque se la considera pago de servicio (costo administrativo y ganancia de las instituciones). De allí que en función del ajuste de los ingresos declarados en la encuesta, el valor de este tipo de prestaciones se compute, tanbién en términos netos, dentro del conjunto de partidas que constituyen las transferencias corrientes.

\section{Ajuste al concepto de Ingreso disponible}

Un punto que puede suscitar debate es el siguiente: si los ingresos de los hogares medidos en la encuesta debieran compatibilizarse a nivel global con el concepto de ingreso disponible de las cuentas nacionales, o bien si determinadas partidas debieran considerarse estrictamente en términos de ingreso bruto. En otras palabras, si a algunas corrientes de ingreso registradas en la cuenta de los hogares debiera descontárseles o no la contrapartida de gasto en que incurren los hogares por el mismo concepto.

En concreto, esto afectaria, entre otros, a los ingresos de capital en efectivo (rentas de la propiedad recibidas menos intereses y otras rentas pagadas), los seguros de riesgos (indemnizaciones recibidas menos primas netas pagadas) y las transferencias corrientes (las recibidas menos las efectuadas).

En los trabajos de la CEPAL se ha optado por utilizar estas variables sin descontar los respectivos gastos, ya que las encuestas apuntan a captar —n el mejor de los casos- el ingreso bruto correspondiente, y no se puede suponer que, pese a ello, los entrevistados declaren dichos ingresos en términos netos.

A la vez, cabe tener presente tres circunstancias adicionales. La primera, de orden estrictamente práctico, es que el conjunto de estas corrientes de ingreso representa una proporción muy baja del ingreso total de los hogares, de modo que cualquiera sea el criterio que se adopte su incidencia en el resultado final es poca. La segunda, más conceptual, es que en los estudios de pobreza lo que interesa evaluar es la magnitud de los recursos de que dispone el hogar, con prescindencia del destino de esos recursos, cuestión esta última que desde un punto de vista normativo - - y especialmente para el caso de los hogares pobres - queda comprendida en las consideraciones propias de la determinación del valor de la línea de pobreza (o sea, de los gastos requeridos para cubrir las necesidades básicas). Y la tercera es que, de conformiđad con la técnica aplicada para corregir la subdeclaración de los ingresos de capital medidos por las encuestas, donde éstos sólo se imputan al quintil de mayores ingresos, el criterio alternativo se reflejaría cuando más en una leve baja de la concentración del ingreso, pero casi no afectaría la medición de la pobreza.

Lo descrito itustra los avances metodologicos alcanzados en materia de evaluación y corrección de los sesgos que afectan a la medición del ingreso en las encuestas de hogares. Pero al mismo tiempo, advierte de la necesidad de seguir mejorando la calidad de estos ajustes, ${ }^{14}$ junto con elevar la confiabilidad de la información proveniente tanto de las encuestas como de las cuentas nacionales (Altimir, 1987).

\footnotetext{
${ }^{14}$ A esto puede contribuir, por ejemplo, el utilizar más ampliamente -en una primera etapa del ajuste de las encuestas de propositos múltiples- la información que suministran las investigaciones sobre presupuestos familiares. Especialmente importante es la posibilidad de ir más allá de la comparación por fuente de ingreso y desagregar el ajuste por ramas de actividad económica o grupos de ocupación.
} 


\section{VI}

\section{Ampliación de la cobertura del concepto de ingreso}

Como ya se mencionó, el hecho de que los gastos de consumo registrados en las encuestas de hogares corresponda exclusivamente a los gastos de consumo privados significa que una parte de la satisfacción de las necesidades básicas no queda reflejada allí cuando los hogares acceden a determinadas transferencias en calidad de bienes y servicios subsidiados total o parcialmente por el Estado.

En principio, la metodología de líneas de pobreza no presenta grandes dificultades conceptuales para ampliar el concepto de ingreso corriente total de los hogares a fin de incluir el acceso efectivo a esos servicios públicos gratuitos o subsidiados, porque al incorporarlos de manera explícita probablemente disminuiría el coeficiente de Engel (elevando, por ende, la línea de pobreza), junto con aumentar proporcionalmente el ingreso de los hogares.

Veamos un ejemplo simple, de un hogar cuyos ingresos se sitúan a nivel de la línea de pobreza. Supongamos que su relación de gasto está dada por:

Gasto en alimentos

Gasto en alimentos + Otros gastos privados

Un aumento de 50 en su gasto total al considerar el consumo de bienes y servicios públicos no alimentarios se traduciría en:

Gasto en alimentos

Gasto total privado + Bienes públicas

Si bien es cierto que con esto aumenta el gasto total del hogar y su coeficiente de gasto en alimentos disminuye a 0.4 , situando la línea de pobreza en 250 , el valor del consumo de bienes y servicios públicos también debe computarse como parte de los ingresos de ese hogar, con lo cual la capacidad de consumo en relación a la línea de pobreza para ese hogar en particular permanece sin variación.

Del ejemplo se deduce que el problema más bien estriba en lo que varía el acceso de los diferentes hogares, en un mismo momento y a lo largo del tiempo, a los bienes y servicios gubernamentales. $O$, por contraste, en la validez del supuesto sobre una relativa estabilidad de la cuantía y distribución de los bienes públicos, del cual se desprende que los hogares toman sus decisiones de gasto con arreglo al sistema institucional vigente y que, en consecuencia, el coeficiente de Engel es adecuado para estimar el costo global del conjunto de sus necesidades.

Sin embargo, la situación es distinta en el plano empírico. Las encuestas no proveen información suficiente sobre el particular. En muy pocos casos - como la encuesta de Caracterización Socioeconómica Nacional (CASEN) de Chile, por ejemplo-n se identifica a los hogares y personas beneficiadas con transferencias monetarias fiscales y se les consulta acerca del monto de esas prestaciones, o bien se registra la información suficiente como para realizar la correspondiente imputación (apoyada con datos de costo, externos a la encuesta).

Desde luego, el problema es aún mayor en lo que toca a las transferencias no monetarias. El desafío en esta esfera es diseñar y lievar a la práctica los instrumentos adecuados para obtener la información a nivel de cada hogar, y asegurar su periodicidad. Esto acarrea el beneficio adicional de aportar antecedentes necesarios para ampliar el análisis de las condiciones de vida y para hacer el seguimiento y la evaluación de determinados programas sociales. 


\section{VII}

\section{Medidas de la pobreza}

La mayoría de los estudios de pobreza que se elaboran en América Latina normalmente entregan resultados relativos a la identificación y cuantificación de los hogares pobres. Vale decir, proporcionan información acerca de la incidencia de la pobreza, desagregada por unidades geográficas o grupos socioeconómicos. Son más escasos, sin embargo, aquellos que cubren otras dimensiones del problema, como las brechas de pobreza (distancia del ingreso de los pobres respecto de la línea de pobreza) y la severidad de la pobreza (qué tan pobres son los pobres). Asimismo, no es del todo usual que se divulguen antecedentes sobre el perfil o las características de los hogares pobres, que son particularmente útiles para las tareas de diagnóstico y el diseño de políticas.

Entre las numerosas medidas de la pobreza que se han propuesto y que permiten enriquecer las posibilidades analíticas de estos estudios se hallan las que dan cuenta de la insuficiencia de ingreso de los pobres, la magnitud del esfuerzo social requerido para superar la pobreza o, por la vía de la descomposición estadística de los índices, la contribución de distintos factores a la evolución de la incidencia agregada de la pobreza (Ravallion, 1992). Desde un punto de vista formal, cada una de estas medidas presenta ventajas y limitaciones.

Sen (1976) señaló dos axiomas principales que debían cumplir los índices de pobreza: i) el axioma de monotonicidad, según el cual una reducción en el ingreso de un hogar pobre (a igualdad de otras condiciones) debiera incrementar el índice de pobreza, y ii) el axioma de transferencia, según el cual una transferencia de ingresos de un hogar pobre a otro más rico (a igualdad de otras condiciones) debiera incrementar el indice de pobreza.

No todos los índices conocidos satisfacen estas condiciones. Es claro, por ejemplo, que la medida de incidencia de la pobreza $(H)$ no tiene ninguna de estas dos propiedades, en tanto que las de brechas de pobreza satisfacen el axjoma de monotonicidad pero no así el de transferencia. En atención a ello, el mismo Sen propuso un índice que supera estas limitaciones:

$$
P(s)=H[I+(I-I) G]
$$

donde: $H=$ incidencia de la pobreza

$I=$ distancia porcentual del ingreso medio de los pobres respecto a la tínea de pobreza

$G=$ coeficiente de Gini de la distribución del ingreso de los pobres.

Como se advierte, en este índice la incorporación del coeficiente de Gini soluciona el problema de que se mantengan inalteradas las medidas $H$ e $I$ cuando se producen transferencias de ingreso entre los pobres. A su vez, $0 \leq P(s) \leq 1$. De allí se deduce que:

$$
\begin{aligned}
& P(s)=H^{*} I \text { si todos los pobres tienen el mis- } \\
& \text { mo ingreso, }
\end{aligned}
$$

Por su parte, Foster, Greer y Thorbecke (1984) tambiên formularon un indice de pobreza, incorporado en una familia de fndices a partir de diferentes valores del parámetro $\alpha$ (que representa algo así como el grado de aversión a la pobreza por parte de la sociedad). Cuando $\alpha=0$ el índice es igual a la incidencia de pobreza $(H)$; con $\alpha=1$, es igual a la brecha de pobreza $(H * D)$, en tanto que cuando $\alpha=2$ el índice representa la distancia media de ingteso de los pobres, al cuadrado, con respecto a la línea de pobreza (FGT). Así:

$$
P(F G T)=(l / n) \sum_{i=1}^{q}\left[\left(z-y_{i}\right) / z\right]^{2}
$$

donde: $n=$ población total

$q=$ total de pobres

$z=$ línea de pobreza

$y_{i}=$ ingreso del $i$-ésimo individuo pobre.

Este índice cumple con los axiomas de monotonicidad y transferencia y además satisface la propiedad aditiva (y de descomposición). Sin embargo, su 
interpretación —en cuanto al significado del fndice en sí y de sus cambios - no resulta del todo clara.

Los ejemplos anteriores sólo pretenden destacar la posibilidad e importancia de complementar el análisis tradicional sobre la magnitud y evolución de la pobreza, con la incorporación de medidas y perfiles que permitan enriquecer el diagnóstico y conocimiento sobre la situación de los sectores pobres. Desde luego, siempre en el marco de las imitaciones que impone la naturaleza muestral de la información y la confiabilidad de los datos de ingreso que corrientemente se utilizan en este tipo de estudios.

\section{VIII}

\section{Las fuentes de información}

Finalmente, algunos comentarios de orden general sobre las fuentes de información que se utilizan para elaborar los estudios de pobreza por el método de la línea de pobreza: específicamente, las encuestas de hogares.

Para nuestros fines cabe distinguir al menos tres clases de encuestas de hogares que se llevan a cabo regularmente en América Latina, y cuya información permite calcular indicadores sociales, incluidos los de pobreza.

En primer lugar están las encuestas de hogares de propósitos múltiples, incorporadas en programas permanentes o de ejecución periódica. Son aquellas que se realizan a lo menos una vez al año, cuyo módulo central se orienta generalmente a la medición del empleo y que al mismo tiempo registran información sobre un conjunto más amplio de características de las personas (demográficas, de migración, educación e ingresos) y de sus viviendas. La mayoría de ellas incluye en algunas de sus rondas módulos especiales que van adosados al cuestionario principal de la encuesta; éstos han pasado a constituir un expediente interesante y de bajo costo que se adapta bien a las necesidades de investigación y profundización de ciertos temas (trabajo femenino, educación y capacitación, acceso a servicios de salud, etc.).

Un segundo tipo de encuestas, que se realizan de manera menos frecuente y con periodicidad variable, está constituido por las encuestas de ingresos y gastos $o$ de presupuestos familiares. También son investigaciones muestrales que aportan antecedentes muy útiles para el examen de la situación social. Normalmente contienen, además de ciertos atributos generales de las personas y de las viviendas, un registro detallado del ingreso y del gasto de las familias, a partir de lo cual se definen las canastas básicas de alimentos cuyo valor se utiliza en la estimación de las líneas de pobreza. A su vez, efectúan un control (estadístico) de la estacionalidad del ingreso y de los gastos si se entrevista a distintos hogares habitualmente a lo largo de todo un año. Esto redunda en información de bastante buena calidad, a la que también contribuyen los instrumentos y procedimientos de captación, los métodos de evaluación (balance ingreso-gasto y verificaciones en lugares de compra) y la selección del entrevistado (en muchos casos informante directo). Además, estas encuestas suelen integrarse en el marco conceptual de las cuentas nacionales, especialmente en lo que respecta a la estructura de consumo de los hogares, y su temática hace posible -en algún grado-el estudio del comportamiento de los gastos de consumo frente al impacto de determinadas políticas económicas de coyuntura. Contra este último objetivo conspiran, sin embargo, lo esporádico de estas investigaciones (debido, entre otras cosas, a su alto costo), su cobertura geografica generalmente subnacional, su alta complejidad y el que, por to mismo, sea dificil contar con muestras tipo panel. Asimismo, su gran extensión hace difícil profundizar en temas como la educación o la vivienda, e incorporar otros como el acceso a los servicios de salud o la recepción de transferencias no monetarias del Estado.

Por último, cabe mencionar las encuestas preparadas especialmente para hacer un seguimiento y evaluación de las condiciones sociales y de las situaciones de pobreza en la región. Estas encuestas, que se efectúan cada dos o tres años, están orientadas a investigar con mayor extensión o profundidad determinados aspectos, como el impacto de las políticas y programas sociales en los diferentes sectores de la población. Un prototipo de ellos, que viene teniendo bastante difusión e influencia en America Latina, es la encuesta de Caracterización Socioeconómica Nacional (CASEN) de Chile. En este tipo de encuestas normalmente se investiga en detalle no sólo los recursos (ingresos) globales del hogar sino también el acceso efectivo a determinados programas asistenciales (alimenta- 
ción escolar, subsidios alimentarios o de vivienda, etc.) y a los servicios públicos (salud, educación, infraestructura, etc.). Esto permite medir el efecto y evaluar el grado de focalización de esos programas, y a la vez analizar importantes características de los grupos beneficiarios a base de información del hogar recogida por la encuesta, y no por los registros administrativos propios de cada programa.

Cabe hacer notar que muchos países de la región cuentan con programas que comprenden dos e incluso los tres tipos de encuestas mencionadas, sujeto cada uno de ellos a periodicidades distintas. Sin embargo, en los últimos tiempos, y por la creciente demanda de información del sector social, han quedado de manifiesto con mayor fuerza ciertas deficiencias, especialmente en la cobertura temática y la periodicidad de las encuestas, así como tambiên en la consistencia de los datos generados por diferentes fuentes y la aptitud de estas últimas para responder a las necesidades impuestas por las políticas y proyectos sociales. Esto ha llevado en algunos casos a la proliferación de encuestas, o bien a ampliar el contenido de los cuestionarios para obtener más información de lo que es razonable esperar del tipo de encuesta e instrumentos utilizados (por

\section{IX}

\section{Conclusión}

Aun con la misma metodología para la medición de la pobreza, en este caso la del ingreso o de líneas de pobreza, es posible que diversos estudios lleguen a resultados diferentes, debido a que su aplicación concreta suele transitar por diversas variantes en cuanto a los criterios, procedimientos y fuentes de información utilizados. Esto provoca un natural desconcierto, socava la credibilidad y confiabilidad técnica de las investigaciones y limita sus posibilidades de use en el campo de la acción social. De alli que sea conveniente identificar los factores que explican tales discrepancias, con miras a avanzar hacia una mayor consistencia y homogeneidad de las mediciones.

En el presente artículo se ha intentado reseñar algunos de los factores cuya incidencia en la identificación de los hogares pobres y en el cálculo de la extensión de la pobreza se estima de gran importancia. Asimismo, se formulan sugerencias que pueden ser útiles para un debate futuro que busque elevar el gra- ejemplo, intentar la investigación de los temas propios de las encuestas de ingresos y gastos mediante los procedimientos más ágiles y baratos, pero menos eficaces, de las encuestas de propósitos múltiples).

Junto con persistir en el esfuerzo por mejorar la calidad de los datos que se producen a través de las diferentes investigaciones, nos parece necesario esclarecer más las potencialidades de cada tipo de encuesta, fomentando cierta especialización, pero en un marco de consistencia y complementación entre ellas (sistema integrado). Así, en aquellos países carentes de una o más de estas encuestas, que son mayoría en la región, debiera avanzarse en completar el sistema. La diversidad de situaciones que exhiben los paises determinará que en algunos casos se apunte a lograr continuidad y mayor calidad en la encuesta de propósitos múttiples, ampliando su cobertura geográfica y fortaleciendo el soporte institucional. En otros, en tanto, cobrará importancia la realización de una nueva encuesta de ingresos y gastos, o bien el diseño y puesta en práctica de una investigación sobre las condiciones de vida de la población. En los más avanzados, por su parte, lo central será mejorar la coherencia e integracín de todos estos instrumentos. do de consenso respecto de la aplicación de conceptos $\mathrm{y}$ métodos. A modo de síntesis, se ofrecen a continuación algunas observaciones finales.

Es habitual que se establezcan costos distintos de la canasta básica de alimentos, debido a diferencias en la información y en los procedimientos que se emplean. Esto sugeriría que en los estudios de pobreza se de biera trabajar más bien con un intervalo de valores y no con un valor único.

La estructura del gasto varía, en función del tamaño y la composicion del hogar y de las etapas del ciclo de vida familiar entre otros factores. De modo que más que aplicar un coeficiente (promedio) de gasto en bienes no alimentarios para todos los hogares de un grupo pertinente de ingresos, debiera tenderse a utilizar "un juego de coeficientes". Asimismo, cuando no se dispone de datos recientes sobre la distribución del gasto, dichos coeficientes debieran actualizarse, al menos de acuerdo con la evolución de los precios re- 
lativos de los alimentos, por un lado, y con la de los demás productos de consumo, por otro.

El uso de valores expresados en términos per cápita es necesario, pero claramente insuficiente. Las importantes diferencias entre los hogares en lo que toca a determinados atributos claves hacen aconsejable evaluar sus necesidades o sus recursos en unidades de equivalencia. Sin embargo, se está lejos de disponer de buenas medidas de esta indole.

Evaluar la calidad de las mediciones del ingreso en las encuestas de hogares es una tarea esencial en las estimaciones de pobreza por el método de líneas de pobreza. Junto con avanzar hacia una mayor cobertura y precisión de los datos primarios, es posible mejorar aún más los procedimientos para corregir y ajustar esas mediciones.
Una parte del consumo, especialmente de los sectores de bajos ingresos, proviene de transferencias del Estado, que no se captan dentro de los gastos privados que investigan las encuestas. Ellas debieran incorporar instrumentos que permitan obtener este tipo de información.

La estratificación de la poblacion de acuerdo a criterios de pobreza abre la posibilidad de elaborar información e indicadores de gran utilidad para conocer el fenómeno y su evolución.

El mejoramiento de la calidad, cobertura y pertinencia de la información que generan las encuestas de hogares sigue siendo - por muchos motivos - un tema crucial. Paralelamente, se debiera avanzar en cada país hacia la conformación de un sistema integrado de encuestas e indicadores sociales.

\section{Bibliografía}

Altimir, O. (1975): Estimaciones de la distribución del ingreso en América Latina por medio de encuestas de hogares y censos de población. Una evaluación de confíabilidad, Santiago de Chile, CEPAL, agosto, mimeo.

(1979): La dimensión de la pobreza en América Latina, Cuadernos de la CEPAL, No 27, Santiago de Chile, CEPAL. Publicación de las Naciones Unidas, $\mathrm{N}^{\circ}$ de venta S.81.II.G.48. (1987): Income distribution statistics in Latin America and their reliability, The Review of Income and Wealth, $\mathrm{N}^{\circ} 2$, New Haven, Conneclicut. Intemational Association for Research in Income and Wealth.

Beccaria, L. y A. Minujin (1993): Sobre la medición de la pobreza: enseñanzas a partir de la experiencia argentina, Buenos $\mathrm{Ai}$ res, mineo.

Boltvinik, J. (1990): Pobreza y necesidades búsicas, Caracas, Programa de las Naciones Unidas para el Desarrollo (PNUD).

CEPAL (1990): Una estimación de la magnitud de la pobreza en Chile, 1987, LCL.599, Santiago de Chite.

(1991): Magnitud de La pobreza en América Latina en los años ochenta, Estudios e informes de la CEPAL, $N_{1}^{\circ} 81$, LC/G.1653-P, Santiago de Chile.

COPLAMAR (Coordinación General del Plan Nacional de Zonas Deprimidas y Grupos Marginados) (1983): Macroeconomia de las necesidades erenciales en México. Siruación atual y perspectivas at año 2000, México, D.F., Siglo XX1 EditoIes, S.A.

Feres, J.C. (1988): Las encuestas de hogares y la medicion del ingreso en América Latina, Estudios de economía, vol. 15,
$N^{\circ}$ 1, Santiago de Chile, Universidad de Chile, Facultad de Ciencias Administrativas.

(1996): La medición del ingreso en la perspectiva de los estudios de pobreża. El caso de la encuesta CASEN de Chile: 1987 a 1994, Documentos sociales $\mathrm{N}^{\circ} 47$, Santiago de Chile, Ministerio de Planificación y Cooperación (Mideplan)/CEPAL, enero.

Feres, J.C. y A. León (1988): Colombia; Estructura del gasto fantiliar en distintos tipos de hogares, Santiago de Chile, CEPAL, abril, mimeo.

Foster, 1., J. Greer y E. Thorbecke (1984); A class of decomposable poverty measures, Econometrica, Journal of the Econometric Society, vol. 52, $\mathrm{N}^{\circ} 3$, Evanston, Illinois, Sociedad de Econometría.

Gerstenfeld, P. (1993): Mediciones de pobreza en Uruguay. Aspectos merudológicos, LCMVD/R.116, Montevideo, Oficina do la CEPAL en Montevideo.

Ravalion, M.(1992): Poverty comparisons. A guide to concepts and methods, Living standards mensurement study working paper, $N^{\circ} 88$, Washington, D.C., Banco Mundial.

Sen, A. (1976): Poverty: An ordinal approach to measurement, Econtometrica, Journal of the Econometric Society, vol, 44 . $\mathrm{N}^{\circ}$ 2, Evanston, Illinois, Sociedad de Econometría.

(1981): Poverty and Famines. An Essay on Entitlement and Deprivation, Oxford, Reino Unido, Organización Intemacio nal del Trabajo (OIT)/Clarendon. 